\title{
Clinical Study \\ Effect of Optimization of Glycaemic Control on Mannan-Binding Lectin in Type 1 Diabetes
}

\author{
Gry Høst Dørflinger, ${ }^{1}$ Charlotte Brink Holt, ${ }^{1,2}$ Steffen Thiel, ${ }^{2}$ Jakob Appel Østergaard, ${ }^{1}$ \\ and Troels Krarup Hansen ${ }^{1}$ \\ ${ }^{1}$ Department of Endocrinology and Internal Medicine, Aarhus University Hospital, 8000 Aarhus, Denmark \\ ${ }^{2}$ Department of Biomedicine, Aarhus University, 8000 Aarhus, Denmark \\ Correspondence should be addressed to Troels Krarup Hansen; tkh@dadlnet.dk
}

Received 9 August 2017; Accepted 26 October 2017; Published 26 November 2017

Academic Editor: Amie D. Brooks

Copyright (c) 2017 Gry Høst Dørflinger et al. This is an open access article distributed under the Creative Commons Attribution License, which permits unrestricted use, distribution, and reproduction in any medium, provided the original work is properly cited.

\begin{abstract}
Objective. Mannan-binding lectin (MBL) concentration in plasma is increased in subjects with type 1 diabetes and associated with increased mortality and risk of diabetic nephropathy. Recent findings show that pancreas transplantation reduces MBL concentration. Whether the increased MBL concentration is reversed by improved glycaemic control remains unknown. We investigated the effects of improved glycaemic control on MBL concentration in patients with type 1 diabetes. Methods. We measured MBL, fructosamine, and $\mathrm{HbA}_{1 \mathrm{c}}$ at baseline and after 6 weeks in 52 type 1 diabetic patients following the change from conventional insulin therapy to insulin pump therapy. Results. After initiation of insulin pump therapy, the total daily insulin dose was significantly reduced (from $51 \pm 18 \mathrm{IE} /$ day to $39 \pm 13 \mathrm{IE} /$ day, $P<0.0001$ ). There was a significant decrease in $\mathrm{HbA}_{1 \mathrm{c}}$ from $8.6 \%$ to $7.7 \%$ (from $70 \mathrm{mmol} / \mathrm{mol}$ to $61 \mathrm{mmol} / \mathrm{mol}, P<0.0001$ ) and in fructosamine levels (from $356 \mu \mathrm{mol} / \mathrm{L}$ to $311 \mu \mathrm{mol} / \mathrm{L}, P<0.0001$ ). MBL levels decreased by $10 \%$ from $2165 \mu \mathrm{g} / \mathrm{L}$ (IQR $919-3389 \mu \mathrm{g} / \mathrm{L}$ ) at baseline to $1928 \mu / \mathrm{L}(\mathrm{IQR} 811-2758 \mu \mathrm{g} / \mathrm{L})$ at follow-up $(P=0.005)$, but MBL change was not significantly correlated with changes in insulin dose, $\mathrm{HbA}_{1 \mathrm{c}}$, or fructosamine. Conclusions. MBL concentration decreased following the initiation of insulin pump therapy in patients with type 1 diabetes and did not correlate with changes in glycaemic control.
\end{abstract}

\section{Introduction}

Diabetic vascular complications progress in part as a consequence of hyperglycaemia. A growing body of evidence links the complement system, in particular mannan-binding lectin (MBL) and the lectin partway, to this pathophysiological process.

MBL is a soluble pattern recognition molecule of the innate immune system that may activate the complement system via the lectin pathway. Concentration of circulating MBL is predominantly determined by the genotype, where polymorphisms give rise to large interindividual differences [1]. Intraindividual differences in MBL concentrations are far smaller [2] and fluctuate as an acute-phase response [3] and from hormonal influence $[4,5]$.
MBL levels are increased in patients with type 1 diabetes, and high levels of MBL have been associated with increased mortality [6] and development of nephropathy [7-9]. Studies in mice have shown that the absence of MBL minimizes the inflammatory injury induced by ischemia [10-12]. Furthermore, deficiency of $\mathrm{MBL}$ protects mice against diabetic nephropathy [13]. On the other hand, low MBL levels have been associated with enhanced risk of myocardial infarction [14] and more pronounced plaque formation [15]. Also, MBL seems to have a beneficial role in the clearance of atherogenic lipoproteins by monocytes and macrophages [16].

MBL levels are increased in type 1 diabetic patients without any association between the genotype and type 1 diabetes $[7,17,18]$, but whether this is reversible by improving glycaemic control remains unknown. Animal studies 
demonstrate a clear association between the increase in blood glucose and an increase in MBL levels following the induction of diabetes [19]. Also, altered MBL concentration was observed following pancreas transplantation in patients with type 1 diabetes [20]. We therefore aimed to investigate the possible association between intensified glycaemic regulation and MBL levels in patients with type 1 diabetes.

\section{Materials and Methods}

We included 52 consecutive patients with type 1 diabetes from our outpatient clinic. The patients were selected for switch from conventional insulin therapy to insulin pump therapy due to poor metabolic control.

Insulin therapy was based on insulin aspart (Novo Nordisk A/S, Bagsværd, Denmark) and administered subcutaneously by a pump system (Minimed 512; Medtronic, Northridge, CA, USA).

Blood samples were collected at baseline and after 6 weeks of treatment with a pump. Serum was isolated from venous blood drawn from the cubital vein and immediately frozen to $-80^{\circ} \mathrm{C}$ until time of analysis.

The study was conducted in accordance with the Helsinki II Declaration and approved by the local Ethics Committee.

2.1. Assays. Serum MBL concentrations were measured using an in-house time-resolved immunofluorometric assay with a lower detection level of $10 \mu \mathrm{g} / \mathrm{L}$ [7]. In brief, microtiter wells were coated with mannan followed by incubation with samples diluted 200-fold. After washing, a monoclonal antiMBL antibody (131-1; Immunolex, Copenhagen, Denmark) labelled with europium using reagents from Wallac Oy (Turku, Finland) was added. After incubation and washing, the amount of the bound europium-labelled antibody was assessed by time-resolved fluorometry (Delphia; Wallac, Turku, Finland). We used two established tests $\left(\mathrm{HbA}_{1 \mathrm{c}}\right.$ and fructosamine) to monitor the effect of treatment. $\mathrm{HbA}_{1 \mathrm{c}}$ is used to a great extent in our outpatient clinic to monitor glycaemic control; however, fructosamine is only used in special cases as in conditions with changed erythrocyte lifespan (e.g., haemolysis or splenectomy).

Fructosamine concentration was estimated by a commercially available kit (ABX Pentra Fructosamine, Montpellier, France) based on the tetrazolium method. $\mathrm{HbA}_{1 \mathrm{c}}$ was measured at the Clinical Biochemistry Department using gold standard methods.

2.2. Statistical Methods. MBL levels were nonnormally distributed, and values were given as medians with interquartile ranges (IQR). All other values were given as means \pm SD. To analyse changes in MBL from baseline to follow-up, we used the Wilcoxon signed-rank test, whereas paired Student's $t$-test was used for normally distributed variables. Spearman correlation with two-tailed probability values was used to estimate the strength of association between the observed changes. Statistical significance was assumed at $P<0.05$. All statistical calculations were performed with IBM SPSS for Windows (version 20; IBM, Armonk, NY, USA).

\section{Results}

The mean age of the participant was $40 \pm 11$ years, with average diabetes duration of $21 \pm 2$ years. After initiation of insulin pump therapy, the patients' total daily insulin dose was significantly reduced (from $51 \pm 18 \mathrm{IE} /$ day to $39 \pm 13 \mathrm{IE} /$ day, $P<0.0001$ ) (Figure 1(a)). Despite this, there was a significant decrease in both $\mathrm{HbA}_{1 \mathrm{c}}$ from $8.6 \%$ to $7.7 \%$ (from $70 \mathrm{mmol} / \mathrm{mol}$ to $61 \mathrm{mmol} / \mathrm{mol}, P<0.0001$ ) (Figure $1(\mathrm{~b})$ ) and fructosamine levels (from $356 \mu \mathrm{mol} / \mathrm{L}$ to $311 \mu \mathrm{mol} / \mathrm{L}$, $P<0.0001$ ) (Figure $1(\mathrm{c})$ ). The relative reductions in $\mathrm{HbA}_{1 \mathrm{c}}$ and fructosamine levels were highly correlated $(\rho=0.45$, $P<0.001)$ but were not correlated with changes in insulin dose $(\rho=0.21, P=0.13$ and $\rho=0.17, P=0.22$, resp.).

There was a $10 \%$ decrease in MBL concentration from $2165 \mu \mathrm{g} / \mathrm{L}$ (IQR $919-3389 \mu \mathrm{g} / \mathrm{L}$ ) at baseline to $1928 \mu \mathrm{g} / \mathrm{L}$ (IQR $811-2758 \mu \mathrm{g} / \mathrm{L})$ at follow-up $(P=0.005)$ (Figure 2). In exploratory analysis, patients were divided into two groups according to the median MBL concentration at baseline $(2165 \mu \mathrm{g} / \mathrm{L})$ as this would indicate either high-expressing MBL genotypes or low-expressing MBL genotypes. In patients with MBL above the median, MBL concentration changed from $3379 \mu \mathrm{g} / \mathrm{L}$ (IQR 2535-4061 $\mu \mathrm{g} / \mathrm{L}$ ) to $2711 \mu \mathrm{g} / \mathrm{L}$ (IQR $2199-3910 \mu \mathrm{g} / \mathrm{L})(P=0.07)$ whereas in patients with $\mathrm{MBL}$ below the median, MBL concentration changed from $955 \mu \mathrm{g} / \mathrm{L}$ (IQR 399-1429) to 877.5 (252-1149) $(P=0.01)$.

The change in $\mathrm{MBL}$ was not significantly correlated with the changes in insulin dose $(\rho=0.08, P=0.58)$, $\mathrm{HbA}_{1 \mathrm{c}}(\rho=-0.15, P=0.29)$, or fructosamine $(\rho=0.09$, $P=0.53)$. Even when the analyses were performed in the subgroup of patients with MBL levels above the median, which would be expected to be carriers of the high-coding MBL genotypes, there were no correlations between changes in $\mathrm{MBL}$ and changes in insulin, $\mathrm{HbA}_{1 \mathrm{c}}$, or fructosamine (data is not shown).

\section{Discussion}

Our main goal was to examine the impact of improved glycaemic regulation on MBL levels in patients with type 1 diabetes. We found a significant decline in $\mathrm{HbA}_{1 \mathrm{c}}$ and fructosamine levels in the patients following the change from conventional insulin therapy to insulin pump therapy. In parallel with this, we observed a significant reduction in MBL levels. This was seen despite a significant decrease in total insulin dose used.

It is well established that MBL levels are increased in patients with diabetes compared with healthy control subjects $[7,9,17,21]$. Animal studies have demonstrated that the rise in MBL is secondary to the induction of diabetes in a streptozotocin model of type 1 diabetes [19]. We originally hypothesized that insulin could have an inhibitory effect on $\mathrm{MBL}$ production in the liver. The higher levels of MBL in patients treated with subcutaneous insulin could thus be due to lower concentrations of insulin in the portal system compared to the portal insulin levels seen in subjects with normal pancreatic insulin secretion [17]. We were, however, not able to demonstrate the inhibitory effect of insulin on MBL production from human hepatocytes in in vitro studies [22]. 


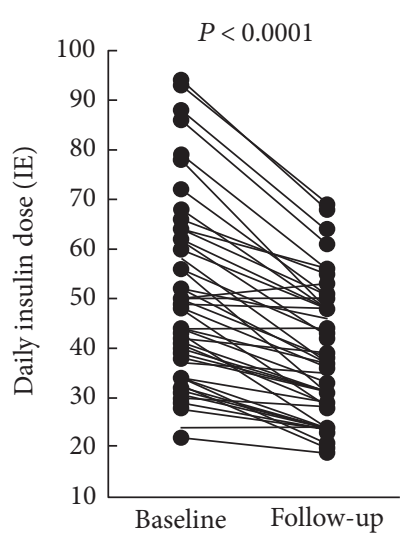

(a)

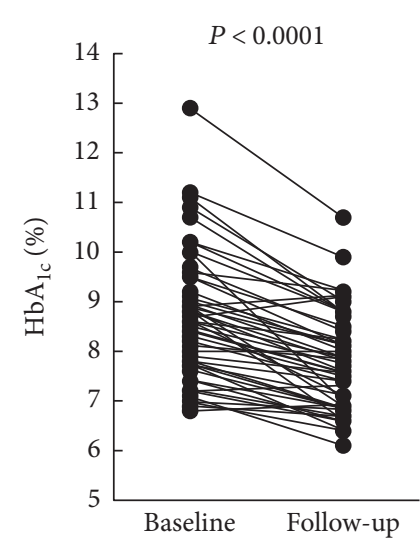

(b)

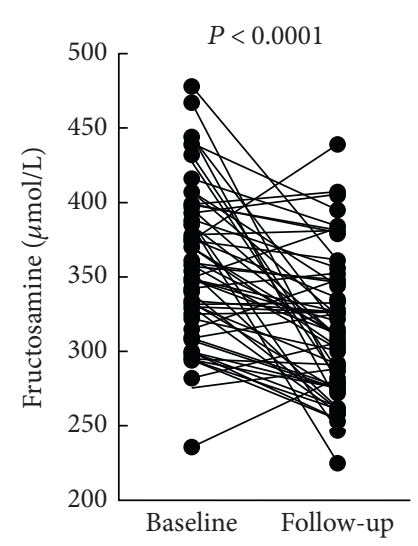

(c)

Figure 1: Daily insulin dose (a), $\mathrm{HbA}_{1 \mathrm{c}}$ (b), and plasma levels of fructosamine (c) at baseline and after 6 weeks in 52 type 1 diabetic patients changed from conventional insulin therapy to insulin pump therapy. $P$ values refer to the change from baseline to follow-up by Student's $t$-test.

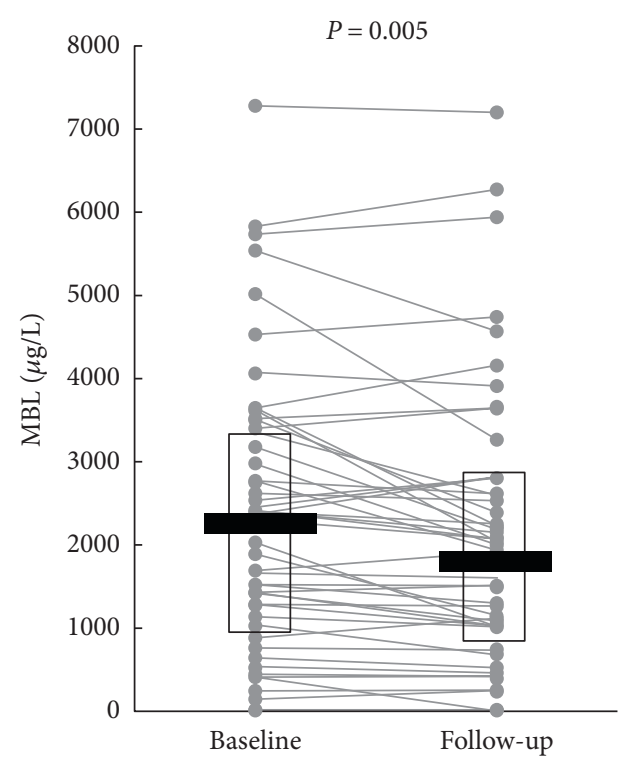

FIGURE 2: Distribution of serum MBL at baseline and after 6 weeks in 52 type 1 diabetic patients changed from conventional insulin therapy to insulin pump therapy. Horizontal bars represent medians, and boxes indicate IQRs. $P$ value refers to the change from baseline to follow-up by the Wilcoxon signed-rank test.

It is a common clinical observation that it is possible to obtain significantly lower $\mathrm{HbA}_{1 \mathrm{c}}$ levels with smaller total insulin dose when using insulin pump therapy compared to conventional basal-bolus therapy. In the present study, MBL levels declined despite the use of less insulin, and without any correlation with changes in $\mathrm{HbA}_{1 \mathrm{c}}$ and fructosamine. This seems to indicate that MBL levels are not affected by glycaemic control per se, but rather by other aspects of the type 1 diabetes pathogenesis. Our small sample size may however introduce risk of statistical type 2 errors in our analysis.

Another interesting way of investigating the effect of glycaemic control on circulating levels of $\mathrm{MBL}$ is through pancreas-kidney transplantation [20]. The authors observed elevated plasma MBL levels in patients with diabetic nephropathy, which were normalized after pancreas-kidney transplantation. Kidney transplantation in solitude did not have a corresponding effect on MBL levels, indicating that glycaemic control is the driving factor.

In a cross-sectional study, Bouwman et al. [21] found that MBL serum levels as well as MBL complex activity were elevated at diagnosis of type 1 diabetes in juvenile subjects with high MBL-producing genotypes compared to their healthy siblings. For all genotypes, they found MBL complex activity, but not MBL serum levels, to correlate with fructosamine concentrations. The authors hypothesize that the elevated MBL serum level was the result of the immunopathogenesis of type 1 diabetes, whereas the elevated MBL complex activity may be affected by glycaemic control.

We found no correlation between $\mathrm{MBL}$ and fructosamine, $\mathrm{HbA}_{1 \mathrm{c}}$, or total insulin dose. A previous study has shown a significant correlation between $\mathrm{HbA}_{1 \mathrm{c}}$ and $\mathrm{MBL}$ in patients with type 1 diabetes, especially among patients with high MBL genotypes [7]. MBL genotypes were not available in the present study. We therefore divided patients according to $\mathrm{MBL}$ concentration either above or below the MBL median as an indicator of high-expression and lowexpression MBL genotypes [17]. However, we still found no correlation between $\mathrm{HbA}_{1 \mathrm{c}}$ and $\mathrm{MBL}$ even in the "high MBL concentration" group.

The regulation of MBL production is probably multifactorial, and the immunopathogenesis of diabetes may to some extent take part. Animal studies indicate that both increased production and prolonged half-life of MBL in diabetes may explain the kinetics of the increase in MBL seen in diabetic animals [19]. Despite the absence of a correlation between $\mathrm{MBL}$ and $\mathrm{HbA}_{1 \mathrm{c}}$ in our study, it is intriguing to consider hyperglycaemia-induced low-grade inflammation a confounding factor in enhanced MBL production, especially in view of the significant parallel fall in $\mathrm{MBL}$ and $\mathrm{HbA}_{1 \mathrm{c}}$ [20]. Furthermore, the relatively short time to follow up of 6 weeks may have disguised a potential correlation. However, using fructosamine to estimate the average level of blood glucose control over a period of 2-3 weeks, we hoped to find a correlation. 
A significant decrease in MBL following intensive insulin treatment has previously been described in an intervention study of 451 critically ill patients [3], and in this study, the intensive insulin treatment was also associated with a significant reduction in inflammation as indicated by reduced high-sensitivity C-reactive protein (hs-CRP) levels [3].

In conclusion, $\mathrm{MBL}$ levels are significantly reduced following the initiation of insulin pump therapy in type 1 diabetic patients, despite a significant reduction in total insulin dose, but the change was not correlated with indicators of improved glycaemic control.

\section{Conflicts of Interest}

The authors declare that there is no conflict of interest regarding the publication of this article.

\section{Acknowledgments}

The study was supported by the Danish Council for Independent Research, the Novo Nordisk, and the Danish Diabetes Association.

\section{References}

[1] R. Steffensen, S. Thiel, K. Varming, C. Jersild, and J. C. Jensenius, "Detection of structural gene mutations and promoter polymorphisms in the mannan-binding lectin (MBL) gene by polymerase chain reaction with sequencespecific primers," Journal of Immunological Methods, vol. 241, no. 1-2, pp. 33-42, 2000.

[2] H. Ytting, I. J. Christensen, S. Thiel et al., "Biological variation in circulating levels of mannan-binding lectin (MBL) and MBL-associated serine protease- 2 and the influence of age, gender and physical exercise," Scandinavian Journal of Immunology, vol. 66, no. 4, pp. 458-464, 2007.

[3] T. K. Hansen, S. Thiel, P. J. Wouters, J. S. Christiansen, and G. van den Berghe, "Intensive insulin therapy exerts antiinflammatory effects in critically ill patients and counteracts the adverse effect of low mannose-binding lectin levels," The Journal of Clinical Endocrinology \& Metabolism, vol. 88, no. 3, pp. 1082-1088, 2003.

[4] A. L. D. Riis, T. K. Hansen, S. Thiel et al., "Thyroid hormone increases mannan-binding lectin levels," European Journal of Endocrinology, vol. 153, no. 5, pp. 643-649, 2005.

[5] T. K. Hansen, S. Thiel, R. Dall et al., "GH strongly affects serum concentrations of mannan-binding lectin: evidence for a new IGF-I independent immunomodulatory effect of GH," The Journal of Clinical Endocrinology \& Metabolism, vol. 86, no. 11, pp. 5383-5388, 2001.

[6] J. A. Ostergaard, S. Thiel, M. Lajer et al., "Increased all-cause mortality in patients with type 1 diabetes and high-expression mannan-binding lectin genotypes: a 12-year follow-up study," Diabetes Care, vol. 38, no. 10, pp. 1898-1903, 2015.

[7] T. K. Hansen, L. Tarnow, S. Thiel et al., "Association between mannose-binding lectin and vascular complications in type 1 diabetes," Diabetes, vol. 53, no. 6, pp. 1570-1576, 2004.

[8] T. K. Hansen, C. Forsblom, M. Saraheimo et al., "Association between mannose-binding lectin, high-sensitivity C-reactive protein and the progression of diabetic nephropathy in type 1 diabetes," Diabetologia, vol. 53, no. 7, pp. 1517-1524, 2010.
[9] P. Hovind, T. K. Hansen, L. Tarnow et al., "Mannose-binding lectin as a predictor of microalbuminuria in type 1 diabetes: an inception cohort study," Diabetes, vol. 54, no. 5, pp. 15231527, 2005.

[10] R. Gesuete, M. Stravalaci, S. Fumagalli et al., "Targeting mannose-binding lectin confers long-lasting protection with a surprisingly wide therapeutic window in cerebral ischemia," Circulation, vol. 126, no. 12, pp. 1484-1494, 2013.

[11] M. Moller-Kristensen, W. Wang, M. Ruseva et al., "Mannanbinding lectin recognizes structures on ischaemic reperfused mouse kidneys and is implicated in tissue injury," Scandinavian Journal of Immunology, vol. 61, no. 5, pp. 426-434, 2005.

[12] M. C. Walsh, T. Bourcier, K. Takahashi et al., "Mannose-binding lectin is a regulator of inflammation that accompanies myocardial ischemia and reperfusion injury," Journal of Immunology, vol. 175, no. 1, pp. 541-546, 2005.

[13] J. Østergaard, S. Thiel, M. Gadjeva, T. K. Hansen, R. Rasch, and A. Flyvbjerg, "Mannose-binding lectin deficiency attenuates renal changes in a streptozotocin-induced model of type 1 diabetes in mice," Diabetologia, vol. 50, no. 7, pp. 15411549, 2007.

[14] I. T. Vengen, H. O. Madsen, P. Garred, C. Platou, L. Vatten, and V. Videm, "Mannose-binding lectin deficiency is associated with myocardial infarction: the HUNT2 study in Norway," PLoS One, vol. 7, no. 7, article e42113, 2012.

[15] R. A. Matthijsen, M. P. J. de Winther, D. Kuipers et al., "Macrophage-specific expression of mannose-binding lectin controls atherosclerosis in low-density lipoprotein receptor-deficient mice," Circulation, vol. 119, no. 16, pp. 2188-2195, 2009.

[16] D. A. Fraser and A. J. Tenner, "Innate immune proteins C1q and mannan-binding lectin enhance clearance of atherogenic lipoproteins by human monocytes and macrophages," Journal of Immunology, vol. 185, no. 7, pp. 3932-3939, 2010.

[17] T. K. Hansen, S. Thiel, S. T. Knudsen et al., "Elevated levels of mannan-binding lectin in patients with type 1 diabetes," The Journal of Clinical Endocrinology \& Metabolism, vol. 88, no. 10, pp. 4857-4861, 2003.

[18] M. A. Kaunisto, L. Sjolind, R. Sallinen et al., "Elevated MBL concentrations are not an indication of association between the MBL2 gene and type 1 diabetes or diabetic nephropathy," Diabetes, vol. 58, no. 7, pp. 1710-1714, 2009.

[19] J. A. Ostergaard, M. Bjerre, F. Dagnaes-Hansen, T. K. Hansen, S. Thiel, and A. Flyvbjerg, "Diabetes-induced changes in mannan-binding lectin levels and complement activation in a mouse model of type 1 diabetes," Scandinavian Journal of Immunology, vol. 77, no. 3, pp. 187-194, 2013.

[20] R. Bijkerk, P. van der Pol, M. Khairoun et al., "Simultaneous pancreas-kidney transplantation in patients with type 1 diabetes reverses elevated MBL levels in association with MBL2 genotype and VEGF expression," Diabetologia, vol. 59, no. 4, pp. 853-858, 2016.

[21] L. H. Bouwman, P. Eerligh, O. T. Terpstra et al., "Elevated levels of mannose-binding lectin at clinical manifestation of type 1 diabetes in juveniles," Diabetes, vol. 54, no. 10 , pp. 3002-3006, 2005.

[22] C. M. Sorensen, T. K. Hansen, R. Steffensen, J. C. Jensenius, and S. Thiel, "Hormonal regulation of mannan-binding lectin synthesis in hepatocytes," Clinical and Experimental Immunology, vol. 145, no. 1, pp. 173-182, 2006. 


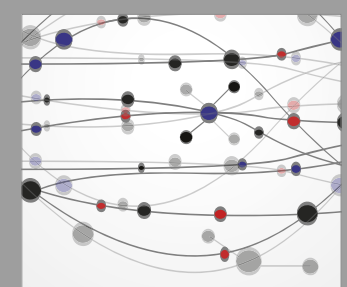

The Scientific World Journal
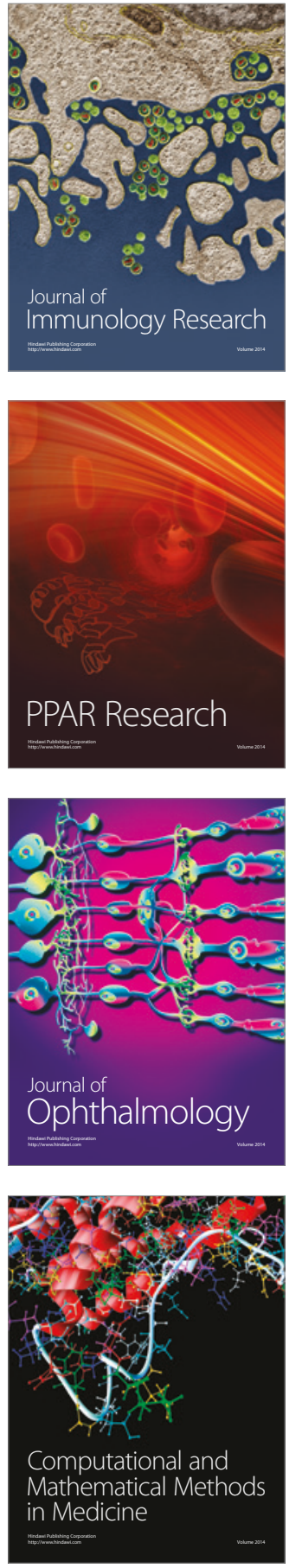

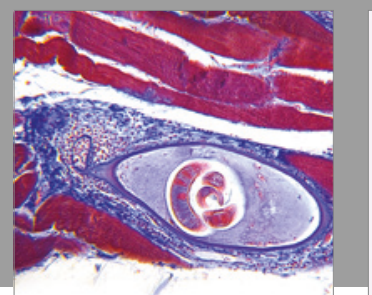

Gastroenterology Research and Practice
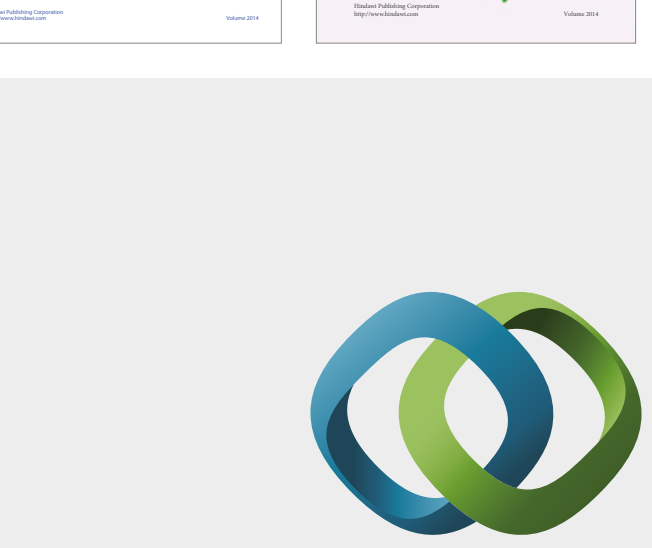

\section{Hindawi}

Submit your manuscripts at

https://www.hindawi.com
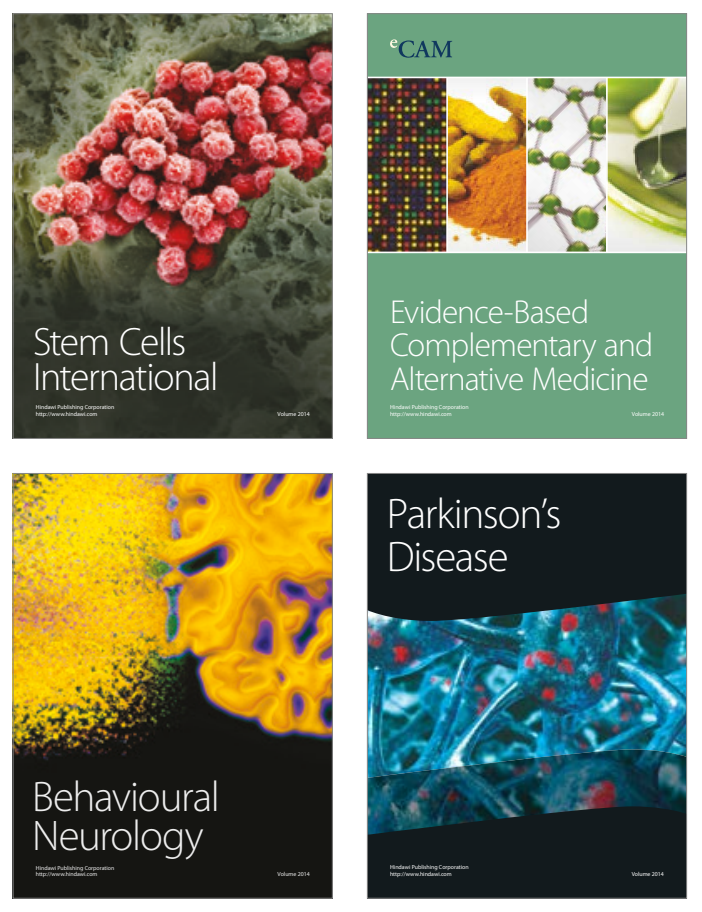
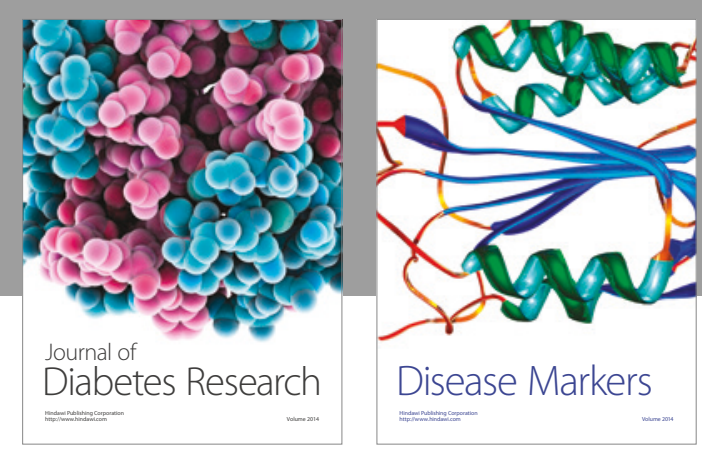

Disease Markers
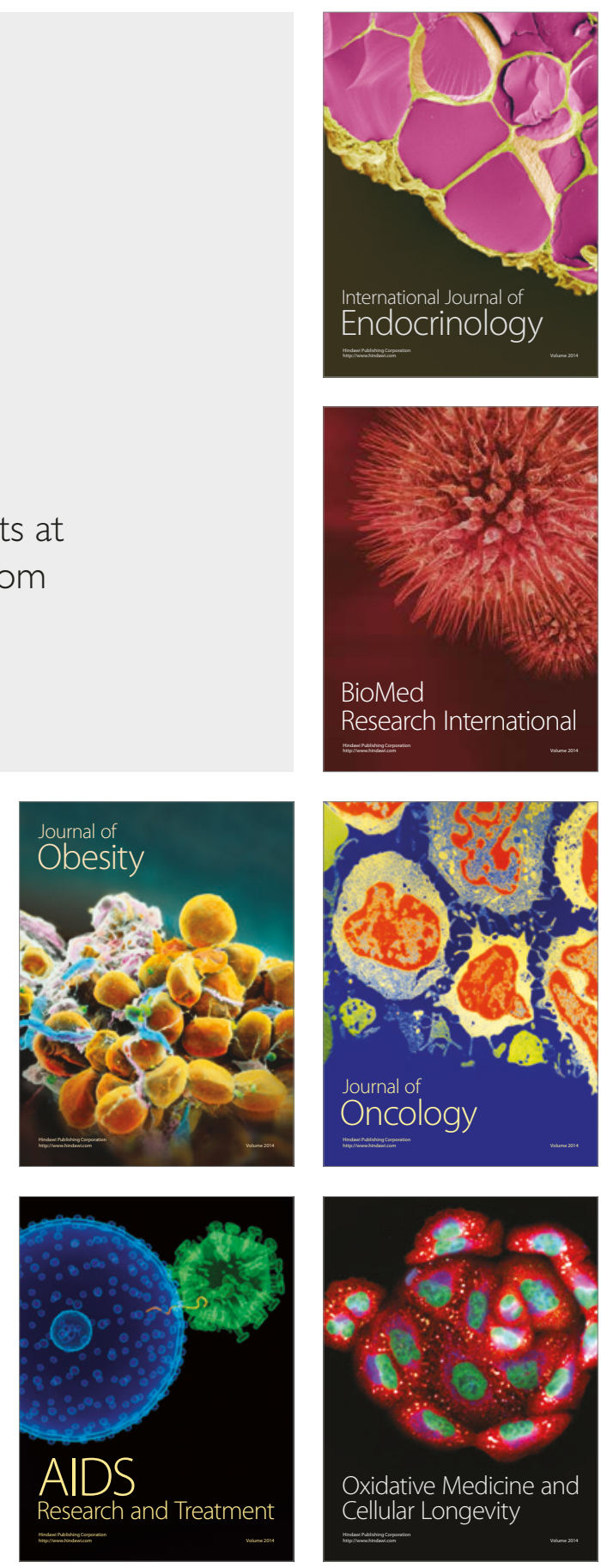